\title{
Comparison of Percutaneous Endoscopic Lumbar Discectomy and Minimally Invasive Transforaminal Lumbar Interbody Fusion for Revision Surgery for the Recurrent Lumbar Disc Herniation After Percutaneous Endoscopic Lumbar Discectomy
}

\author{
Anqi Wang \\ Peking University First Hospital \\ Zhengrong Yu ( $\square$ yuzronline@163.com ) \\ Peking University First Hospital
}

Research article

Keywords: Recurrent lumbar disc herniation, revision surgery, percutaneous endoscopic lumbar discectomy, minimally invasive transforaminal lumbar interbody fusion.

Posted Date: August 5th, 2020

DOI: https://doi.org/10.21203/rs.3.rs-52606/v1

License: (c) (i) This work is licensed under a Creative Commons Attribution 4.0 International License. Read Full License

Version of Record: A version of this preprint was published at Therapeutics and Clinical Risk Management on December 1st, 2020. See the published version at https://doi.org/10.2147/TCRM.S283652. 


\section{Abstract}

Background: Percutaneous endoscopic lumbar discectomy (PELD) has become a feasible alternative to the conventional open surgery for the treatment of lumbar disc herniation (LDH), but PELD is associated with a certain rate of recurrence. Therefore, a good choice of surgical approach for recurrent lumbar disc herniation (rLDH) is important. The purpose of this study was to compare the outcomes between PELD and minimally invasive transforaminal lumbar interbody fusion (MIS-TLIF) for revision surgery for the rLDH after primary PELD surgery.

Methods: This study retrospectively assessed 46 patients who suffered from rLDH after PELD surgery in Peking University First Hospital from January 2015 to June 2019, and patients were divided into two groups, PELD group ( $n=24)$ and MIS-TLIF group $(n=22)$, according to different revision surgical methods. Preoperative data, perioperative conditions, complications, recurrence condition, and clinical outcomes were compared between these two groups.

Results: Compared with the MIS-TLIF group, the PELD group was associated with shorter operative time, less intraoperative hemorrhage, and shorter post-operative hospitalization, but higher recurrence rate. Complication rates were comparable between two groups. Both the two groups could provide patients with satisfactory clinical outcomes at the 12-month follow-up after revision surgery. Compared with MISTLIF, PELD was associated with visual Analog Scale (VAS) scores of back pain, and Oswestry disability index (ODI) at the 1-month follow-up, whereas this effect was equalized after 6 months postoperatively.

Conclusions: Either PELD or MIS-TLIF surgery could provide satisfactory clinical outcomes for revision surgery for the rLDH after primary PELD. PELD brought advantages in shorter operative time, less intraoperative hemorrhage, and shorter post-operative hospitalization over MIS-TLIF, however, higher postoperative recurrence rate of PELD couldn't be ignored. Surgeons must weigh advantages against disadvantages of these surgical methods and fully informed patients preoperatively.

\section{Background}

Lumbar disc herniation (LDH), with a reported prevalence of $1-3 \%$, is a prevalent degenerative disease leading to spinal surgery[1]. Among the operative methods used for lumbar discectomy, the microdiscectomy technique is the gold standard procedure for symptomatic LDH that has not improved with conservative treatment $[2,3]$. With the development of high- resolution endoscopes and corresponding instruments, percutaneous endoscopic lumbar discectomy (PELD) was introduced and has become a feasible alternative to the conventional open surgery for the treatment of LDH[4].

PELD is associated with improvement in back pain and radicular or cauda equine syndromes caused by LDH, which translates to improvement in patients' life quality [5-7]. Despite the advantages of minimal invasiveness, shorter hospital stays, faster rehabilitation, and earlier return to work[7-12], the occurrence of recurrent LDH (rLDH) postoperatively has raised the concerns of many medical researchers[13-15]. The rLDH was usually defined as the same level disc herniation after primary discectomy with a more 
than one month pain-free interval[16]. Yin S et al[17] demonstrated PELD is associated with a certain rate of recurrence (3.6\%), which usually occurred within 6 months postoperatively. Leven et al[18] reported $69 \%$ patient underwent reoperation because of rLDH.

Minimally invasive transforaminal lumbar interbody fusion (MIS-TLIF), first reported by Foley in 2003[19], is a lumbar fusion surgery for LDH which can decrease trauma to back muscles and damage to bony structures [20-25]. As mentioned above, PELD is an effective nonfusion surgery for LDH with the merit of minimal invasiveness as well. Hence, MIS-TLIF and PELD are 2 well-accepted choices for revision surgery for rLDH in the condition of proper indications[26-29]. In this study, we compared the outcomes of these 2 minimally invasive spine surgery methods that are applied to treat the rLDH after primary PELD, and sought to explore some helpful insights into the preoperative selection of the revision surgery for the recurrence of PELD.

\section{Material And Methods}

\section{Research subjects and grouping}

A total of 1536 patients, who received PELD in our hospital from January 2015 to June 2019. Fifty patients who suffered from PELD recurrence and underwent reoperation (PELD or MIS-TLIF surgery) were enrolled in the study, and the retrospective analysis was performed. Before operation, surgeon presented a detailed written informed consent to the patients. This study was approved by the Human Subjects Institutional Review Board at Peking University First Hospital. Patient outcomes were collected independently from participants with informed written consent, and data were analyzed blindly.

The inclusion criteria were as follows: patients who (1) underwent a successful PELD surgery which could be confirmed by a pain-free interval of at least 1 month, (2) recurrent pain symptoms and a magnetic resonance imaging (MRI) confirmation of a herniated fragment on the same level as the previous PELD surgery was achieved, and (3) conservative therapy failed to relieve the recurrent pain.

Exclusion criteria were as follows: patients who (1) the recurrent pain symptoms and MRI confirmation of residual disc fragment occurring within 1 month after the PELD surgery, which is defined as surgical failure, and (2) the herniated disc fragment did not appear at the same level as that in the previous PELD surgery.

In consideration of that the scar formation exists in patients receiving reoperation, to exclude scar formation from real rLDH, all the enrolled patients should exhibit the following characteristics: (1) definite neurological symptom; (2) space-occupying lesions in lumbar spinal canal that were confirmed by MRI; (3) the herniation of nucleus pulposus were observed intraoperatively. Also, the patients with vertebral instability or spondylolisthesis who preferred MIS-TLIF were excluded[14].

Based on these inclusion and exclusion criteria, 46 cases of 50 patients were involved in the following retrospective analysis, each of included patients was appropriate for each of these 2 surgical methods. 
The 46 patients were divided into the following two groups: PELD group $(n=24)$ and MIS-TLIF group $(n=22)$. Patients were fully informed of the details regarding these two surgical methods, including the surgical procedures, experience of the surgeons, complications and recurrence, total cost, and the final choices were made by the patients.

\section{Surgical technique and postoperative management}

Surgeries of patients in the two groups were performed by the same experienced surgeon.

PELD group: PELD was performed as described by Hoogland et al.[30] and $\mathrm{H}$. Michael Mayer and Mario Brock[4]. The procedure was performed under local anesthesia with patients in the prone position on a radiolucent table. The patients were informed of all the procedure steps before beginning the procedure. The patients communicated with the surgeon throughout the entire procedure. After infiltration of the entry point (8-12 cm from the midline) with local anesthetics, an 18-gauge spinal needle was introduced under fluoroscopic guidance. The final target point of the spinal needle was the medial pedicular line on the anteroposterior image and the posterior vertebral line on the lateral image. The subsequent steps were as follows: (1) a guide wire was inserted through the spinal needle; (2) the spinal needle was removed; (3) a small skin incision was created at the entry point; (4) a tapered cannulated obturator was inserted along the guide wire; (5) after contacting the annulus, the obturator was inserted into the disc with hammering, and the timing of foraminoplasty was selected based on the type of prolapse of the disc; and (6) a bevelended, oval-shaped working cannula was inserted into the disc along the obturator. The obturator was removed, and an endoscope was inserted through the cannula. The herniated disc was removed using small forceps. A targeted fragmentectomy was performed in all cases. After the herniated fragment was completely removed, the endoscope was also removed, and a sterile dressing was applied with a 1-point suture (Fig. 1).

MIS-TLIF group: MIS-TLIF was performed as described by Foley et al [19]. All the participants received general anesthesia before MIS-TLIF surgery. C-arm machine and Quadrant System were prepared before the operation. The patient was placed in a prone position on a radiolucent operating table. Under $\mathrm{C}$-arm fluoroscopy, the targeted level was confirmed according to our self-made locator. Based on the spatial relationship, the intervertebral spaces and the pedicle positions were marked on the body surface. An incision was planned by connecting a line between the outer portions of both ends pedicles (approximately $3.0 \mathrm{~cm}$ off midline). Then a skin incision about 2.0 to $3.0 \mathrm{~cm}$ was made on the more symptomatic side or more severe pathology side according to the imaging. The paravertebral muscles were split and retracted laterally to the outer edge of the facet joint, and the zygapophysis was confirmed. Expansion tube was then inserted and Quadrant System was placed. X-ray examination was repeated to confirm the target segments and the placement of Quadrant System. We conducted the decompression by cutting the inferior portion of the lamina, hypertrophied superior and inferior articular processes, and ligamenta flava. Then we enlarged the intervertebral space and appropriate bullet-type interbody cage (Medtronic Sofamor Danek, Memphis, TN 38132, USA) filled with autologous bone was packed into the center of intervertebral space. Following these, Ipsilateral percutaneous pedicle screws (Medtronic 
Sofamor Danek, Memphis, TN 38132, USA) were inserted through the same skin incision, and contralateral percutaneous pedicle screw was also placed through mirror incision under fluoroscopic guidance, also titanium rods (Medtronic Sofamor Danek, Memphis, TN 38132, USA) were inserted and tightened on both sides (Fig. 2). Finally, hemostasis and proper irrigation of the incision was performed and negative pressure drainage was placed followed by closure in layers.

For all the patients in the two groups, off-bed activities could be performed on the second day after the surgery under the protection of waist support. The drain removal was conducted when the drainage fluid for $24 \mathrm{~h}$ was less than $30 \mathrm{ml}[23]$ in MIS-TLIF group. The patients could return to non-manual work or school 2 weeks postoperatively and they were allowed to perform full activity 3 months after the surgery. We encourage sufficient bed-rest and off-bed activities under the protection of waist support within 3 months after surgery.

\section{Clinical assessment}

The preoperative data from all the enrolled patients were assessed in terms of demographic data (age, gender, body mass index [BMI], marital status, alcohol use history, and smoking history) and clinical data (herniation level, paramedian/central herniation, migrated/nonmigrated herniation, and Modic change).

The perioperative conditions (operation time, intraoperative hemorrhage, and postoperative hospitalization), complications, and recurrence condition also were collected and evaluated.

Clinical outcomes were analyzed based on the improvement of back and leg pain, disability, and clinically satisfactory rate. The intensity of back and leg pain were evaluated using visual analog scores (VAS) preoperatively and 1 months, 6 months, 12 months postoperatively. Disability was assessed using the Oswestry disability index (ODI) version 2.0 preoperatively and 1 months, 6 months, 12 months after surgery. The clinically satisfactory was assessed 12 months postoperatively by an independent surgeon using the MacNab criteria[31]. It was defined that excellent outcome as there is no pain and no limitation of normal life; good outcome as there is occasional pain or paresthesia, but no need medication, and no limitation of normal life; fair outcome as pain is somewhat improved but needs medication, and some limitation of normal life; poor outcome as no improvement or worsening, additional operation is needed due to incomplete decompression, development of instability. Excellent and good outcomes were rated as clinically satisfactory.

\section{Statistical Analysis}

Data were analyzed by Statistical package for program (Version12, SPSS, Chicago, IL). The statistic was demonstrated as mean \pm SD. Independent student $t$-test was used to compare the difference of continuous variables between the two groups. Chi-square test was used to compare the difference of dichotomous variables between the two groups. These two tests were used to compare the baseline data and observation parameters between the 2 groups. $P<0.05$ was regarded as statistical significance. 


\section{Results}

\section{Preoperative data of the patients}

All the enrolled patients received one-segment PELD or MIS-TLIF surgery and they were followed up for at least 12 months. The preoperative demographic and clinical characteristics were not significantly different between the 2 groups (Table 1 ).

\section{Perioperative outcomes, complications, and recurrence condition}

Compared with MIS-TLIF group, PELD group was associated with shorter operative time, less intraoperative hemorrhage, and shorter post-operative hospitalization (Table 2).

Complications occurred in 2 patients (8.33 \%) in PELD group and 1 patients (4.55\%) in MIS-TLIF group (Table 2). One case in MIS-TLIF group experienced epidural hematoma, and evacuation of hematoma was performed to the patient who then obtained ideal recovery. One case in PELD group complained of headache during the operation process, which were improved after 1 day after surgery with a sufficient bed rest. In addition, one case in PELD group complained of dysesthesia on the posterior thigh, which were improved with oral intake of neurotrophic drugs for a week after surgery.

The patients in PELD group showed significantly higher recurrence rate (20.83\%) than those in MIS-TLIF group $(0 \%, \mathrm{P}=0.02)$ within 12 months after surgery (Table 2$)$. For the patients suffering recurrence, the symptoms were managed with conservative treatment which consisted of bed-rest and drug therapy (Neurotrophic drugs, and non-steroidal anti-inflammatory drugs). Three of the 5 patients in PELD group with re-recurrence felt that the pain symptom was remitted until the time of last follow up. The other 2 patients received reoperation (MIS-TLIF), and the pain symptom had been obviously relieved until the time of last follow up.

\section{Clinical outcomes}

VAS scores for back and leg pain, and ODI were significantly improved in both groups 1, 6 and 12 months after operation compared with preoperative time. There were no differences in average VAS scores for back and leg pain, and ODI between the two groups at preoperative time, and 6,12 months after operation ( $P>0.05)$. However, 1 months after operation, VAS scores for back pain, and ODI in PELD group were lower than that in MIS-TLIF group $(P<0.05$, Table 3$)$.

According to the MacNab criteria, satisfactory (excellent or good) results were distributed as follows: 87.5\% (21/24) in the PELD group; and 90.9\% (20/22) in the MIS-TLIF group. There was no significant difference in satisfactory rate between these two groups $(P=0.92$, Table 3$)$.

\section{Discussion}


With the development of endoscopic surgical devices, the indications of spine endoscopy have broadened[32, 33], and PELD has been increasingly popular over the last decade for LDH. However, PELD is a nonfusion surgery, and rLDH can occur. Hence, many researchers have focused on the causes of rLDH after PELD surgery. Age, BMI, Modic change, disc height, spinal canal occupancy, postoperative instability or hypermobility, smoking were the factors that demonstrated relative to rLDH after PELD surgery $[13-15,17,34,35]$. These factors may provide the surgeon with a reference in choosing better surgical strategy and evaluation of prognosis. Even though, except for patients with LDH combined with vertebral instability or spondylolisthesis more suitable for fusion surgery, other patients with LDH were fully informed of the details regarding fusion and nonfusion surgical methods, and the final choices were made by the patients. With the advantages of decreased trauma to back muscles and damage to bony structures, reduced intraoperative hemorrhage, shorter hospitalization, faster recovery compared with conventional TLIF and open discectomy, PELD and MIS-TLIF are considered as good alternatives [8, 10, $11,20-25,36]$. In our department, as well PELD and MIS-TLIF are the most common choice of revision surgery for PELD recurrence. In this study, we compared the outcomes of PELD and MIS-TLIF for patients with PELD recurrence and expected to explore some useful insights into the preoperative selection.

In this study, PELD group was associated with shorter operative time, less intraoperative hemorrhage, and shorter post-operative hospitalization than MIS-TLIF group, these perioperative outcomes were consistent with the previous studies[37, 38]. Shorter operative time in PELD group might result from more expansive visual scope and simple operating steps than MIS-TLIF surgery. Less intraoperative hemorrhage and shorter post-operative hospitalization in PELD surgery might benefit from less muscle and bone damage, and shorter operative time, which contributed to faster recovery compared with MIS-TLIF surgery. These outcomes might influence the patients' duration of disability and mental health, also the obedience and trust to the surgeon.

Our data showed that PELD and MIS-TLIF surgeries similarly improved the back and leg pain VAS scores, also reduced ODI 12 months compared to preoperative baselines, and most patients in the two groups feedback clinical satisfactory according to the MacNab criteria, which demonstrates the effectiveness of these two procedures for rLDH revision surgery. These generally favorable outcomes were similar to those reported previously[37-39]. However, back pain VAS scores, ODI in PELD surgery decreased more quickly than MIS-TLIF 1 months after operation, which indicated PELD surgery could remit back and leg pain, and improve quality of life during short-term postoperative time. This was highlighted by the reason of reduced neural tissue retraction, also decreased trauma to paravertebral muscles in discectomy surgery compared with interbody fusion surgery[37, 38].

Complications are nightmares for spine surgeons, especially for re-operation. In our study, one case in MIS-TLIF group experienced epidural hematoma, this patient who had epidural hematoma received unilateral approach for bilateral decompression with MIS-TLIF surgery. Than K.D. et al[40] reported that unilateral approach for bilateral decompression with MIS-TLIF increased the risks of dural laceration and cerebrospinal fluid leak. Similarly, postoperative epidural hematoma might attribute to restricted intraoperative view, tissue or scar adhesion around spinal dural sac, insufficient hemostasis, and 
postoperative obstructed drainage. In addition, one case complained of headache and one case complained of dysesthesia on the posterior thigh in PELD group. The working sheath might compress the nerve root during the process of surgery[41], leading to radicular symptoms after surgery. Sairyo et al. reported that if the endoscopic maneuver duration was too long, the intracranial pressure might increase[42], resulting to headache symptom. Because of distorted anatomy, altered landmarks, and scar formation, revision spine surgery is more challenging than the primary spine surgery and associated with a relatively higher complication rate, especially nerve root injury, dural laceration, and surgical site infection $[43,44]$. Also, the steep learning curves of either MIS-TLIF or PELD need to be considered[4547], expert knowledge of spine anatomy and experience accumulation are required for surgeons to avoid these complications in the future.

Recurrences are also difficult to accept after re-operation. In our study, there were 5 patients in the PELD group and no patient in the MIS-TLIF group suffering from rLDH after revision surgery. The recurrence rate among the patients in the MIS-TLIF group was significantly lower than that in the PELD group. As mentioned above, some common risk factors, for instance old age, obesity, Modic change, were reported predictive of rLDH after PELD surgery. Those 5 participants who experienced recurrence were either old (age > 60 years old) or obese $(\mathrm{BMI}>25)$, and they were at high risk for recurrent herniation after PELD surgery. The risk factors for recurrence after the primary surgery might also predict recurrence after the revision surgery. Additionally, after the primary PELD surgery, the artificial incision in the annulus fibrosus could alter the interlaminar shear stress and make the residual nucleus pulposus more prone to prolapse[48]. Hence, for patients with high risk factors of rLDH, the surgeons should completely inform them about the fusion surgery option and risk for recurrent herniation after either primary or revised nonfusion surgery.

The present study has some limitations. First, it is a retrospective study with small sample size and relatively short follow-up period, an additional multicenter and prospective study with a large sample is needed to confirm the long-term clinical and radiographic outcomes, also more observation parameters such as adjacent degeneration, stability of lumbar spine need to be compared in patients who got different surgeries. A larger sample size would be required to increase the accuracy of the complications and recurrence rates. Second, surgical methods selection in this study has some limitations, more surgical methods such as micro-endoscopic discectomy (MED), open transforaminal lumbar interbody fusion (O-TLIF), posterior lumbar intervertebral fusion (PLIF) and so on for rLDH revision surgery need to be compared in future studies. Third, all operations were done by a single experienced surgeon, so there may be bias resulted from learning curves of different surgeons.

\section{Conclusion}

This study compared the outcomes of PELD and MIS-TLIF for the revision surgery of PELD recurrence. PELD brought advantages in none-general anesthesia, shorter operative time, less intraoperative hemorrhage, and shorter post-operative hospitalization over MIS-TLIF, however, PELD was also associated with higher recurrence rate. PELD surgery could also remit back pain and improve the quality 
of life more quickly during the early stage after revision surgery than MIS-TLIF surgery, but both surgical methods could provide the patients with satisfactory outcomes. In evaluating a patient for revision surgery for PELD recurrence, the advantages of these two surgical methods must be carefully balanced against the disadvantages, and the patients should be completely informed.

\section{Abbreviations}

LDH: lumbar disc herniation; rLDH: recurrent lumbar disc herniation; PELD: percutaneous endoscopic lumbar discectomy; MIS-TLIF: minimally invasive transforaminal lumbar interbody fusion; MRI: magnetic resonance imaging; BMI: body mass index; VAS: visual analog scale; ODI: Oswestry disability index; MED: micro-endoscopic discectomy; 0-TLIF: open transforaminal lumbar interbody fusion; PLIF: posterior lumbar intervertebral fusion.

\section{Declarations}

\section{Acknowledgements}

Not applicable.

\section{Authors' contributions}

ZY designed the study and performed the operations. AW and ZY were responsible for the statistical analysis. AW wrote the paper and ZY made comments, suggested appropriate modifications, and made corrections. All authors read and approved the final version of the manuscript submitted for publication.

\section{Funding}

Not applicable.

\section{Availability of data and material}

The datasets generated/analyzed during the current study are available.

\section{Ethics approval and consent to participate}

This study was approved by the Human Subjects Institutional Review Board at Peking University First Hospital. Participate outcomes were collected independently from participants with written informed consent.

\section{Consent for publication}

All authors read the final manuscript and approved for publication.

\section{Competing interests}


The authors declare that they have no competing interests.

\section{References}

1. Frymoyer JW. Lumbar disk disease: epidemiology. Instr Course Lect 1992;41:217-23.

2. Yeung AT, Tsou PM. Posterolateral endoscopic excision for lumbar disc herniation: Surgical technique, outcome, and complications in 307 consecutive cases. Spine (Phila Pa 1976) 2002;27(7):722-31.

3. Hamawandi SA, Sulaiman, II, Al-Humairi AK. Open fenestration discectomy versus microscopic fenestration discectomy for lumbar disc herniation: a randomized controlled trial. BMC Musculoskelet Disord 2020;21(1):384.

4. Mayer HM, Brock M. Percutaneous endoscopic lumbar discectomy (PELD). Neurosurg Rev 1993;16(2):115-20.

5. Chen C, Fan P, Huang L, Zhen H, Liu L, Wang Y. Percutaneous Endoscopic Lumbar Discectomy as an Emergent Surgery for Cauda Equina Syndrome Caused by Lumbar Disc Herniation. Pain Physician 2020;23(3):E259-E64.

6. Li X, Dou Q, Hu S, Liu J, Kong Q, Zeng J, et al. Treatment of cauda equina syndrome caused by lumbar disc herniation with percutaneous endoscopic lumbar discectomy. Acta Neurol Belg 2016;116(2):185-90.

7. Peng CW, Yeo W, Tan SB. Percutaneous endoscopic lumbar discectomy: clinical and quality of life outcomes with a minimum 2 year follow-up. J Orthop Surg Res 2009;4:20.

8. Ruan W, Feng F, Liu Z, Xie J, Cai L, Ping A. Comparison of percutaneous endoscopic lumbar discectomy versus open lumbar microdiscectomy for lumbar disc herniation: A meta-analysis. Int $J$ Surg 2016;31:86-92.

9. Cao J, Huang W, Wu T, Jia J, Cheng X. Percutaneous endoscopic lumbar discectomy for lumbar disc herniation as day surgery - short-term clinical results of 235 consecutive cases. Medicine (Baltimore) 2019;98(49):e18064.

10. Kim M, Lee S, Kim HS, Park S, Shim SY, Lim DJ. A Comparison of Percutaneous Endoscopic Lumbar Discectomy and Open Lumbar Microdiscectomy for Lumbar Disc Herniation in the Korean: A MetaAnalysis. Biomed Res Int 2018;2018:9073460.

11. Qin R, Liu B, Hao J, Zhou P, Yao Y, Zhang F, et al. Percutaneous Endoscopic Lumbar Discectomy Versus Posterior Open Lumbar Microdiscectomy for the Treatment of Symptomatic Lumbar Disc Herniation: A Systemic Review and Meta-Analysis. World Neurosurg 2018;120:352-62.

12. Ahn SS, Kim SH, Kim DW, Lee BH. Comparison of Outcomes of Percutaneous Endoscopic Lumbar Discectomy and Open Lumbar Microdiscectomy for Young Adults: A Retrospective Matched Cohort Study. World Neurosurg 2016;86:250-8.

13. Kim HS, You JD, Ju Cl. Predictive Scoring and Risk Factors of Early Recurrence after Percutaneous Endoscopic Lumbar Discectomy. Biomed Res Int 2019;2019:6492675. 
14. Wang Y, Ning C, Xu F, Xiang Y, Yao L, Liu Y, et al. Recurrent lumbar disc herniation recurrence after percutaneous endoscopic lumbar discectomy: A case report. Medicine (Baltimore) 2018;97(34):e11909.

15. Kim JM, Lee SH, Ahn Y, Yoon DH, Lee CD, Lim ST. Recurrence after successful percutaneous endoscopic lumbar discectomy. Minim Invasive Neurosurg 2007;50(2):82-5.

16. Yaman ME, Kazanci A, Yaman ND, Bas F, Ayberk G. Factors that influence recurrent lumbar disc herniation. Hong Kong Med J 2017;23(3):258-63.

17. Yin S, Du H, Yang W, Duan C, Feng C, Tao H. Prevalence of Recurrent Herniation Following Percutaneous Endoscopic Lumbar Discectomy: A Meta-Analysis. Pain Physician 2018;21(4):337-50.

18. Leven D, Passias PG, Errico TJ, Lafage V, Bianco K, Lee A, et al. Risk Factors for Reoperation in Patients Treated Surgically for Intervertebral Disc Herniation: A Subanalysis of Eight-Year SPORT Data. J Bone Joint Surg Am 2015;97(16):1316-25.

19. Kambin P. Re: Foley KT, Holly LT, Schwender JD. Minimally invasive lumbar fusion. Spine 2003;28:S26-35. Spine (Phila Pa 1976) 2004;29(5):598-9.

20. Sheng SR, Geng YB, Zhou KL, Wu AM, Wang XY, Ni WF. Minimally invasive surgery for degenerative spondylolisthesis: transforaminal or oblique lumbar interbody fusion. J Comp Eff Res 2020;9(1):4551.

21. Chen YC, Zhang L, Li EN, Ding LX, Zhang GA, Hou Y, et al. An updated meta-analysis of clinical outcomes comparing minimally invasive with open transforaminal lumbar interbody fusion in patients with degenerative lumbar diseases. Medicine (Baltimore) 2019;98(43):e17420.

22. Lin GX, Park CK, Hur JW, Kim JS. Time Course Observation of Outcomes between Minimally Invasive Transforaminal Lumbar Interbody Fusion and Posterior Lumbar Interbody Fusion. Neurol Med Chir (Tokyo) 2019;59(6):222-30.

23. Yang Y, Liu ZY, Zhang LM, Pang M, Chhantyal K, Wu WB, et al. Microendoscopy-Assisted Minimally Invasive Versus Open Transforaminal Lumbar Interbody Fusion for Lumbar Degenerative Diseases: 5-Year Outcomes. World Neurosurg 2018;116:e602-e10.

24. Karikari IO, Isaacs RE. Minimally invasive transforaminal lumbar interbody fusion: a review of techniques and outcomes. Spine (Phila Pa 1976) 2010;35(26 Suppl):S294-301.

25. Parker SL, Adogwa O, Witham TF, Aaronson OS, Cheng J, McGirt MJ. Post-operative infection after minimally invasive versus open transforaminal lumbar interbody fusion (TLIF): literature review and cost analysis. Minim Invasive Neurosurg 2011;54(1):33-7.

26. Ahn Y, Lee SH, Park WM, Lee HY, Shin SW, Kang HY. Percutaneous endoscopic lumbar discectomy for recurrent disc herniation: surgical technique, outcome, and prognostic factors of 43 consecutive cases. Spine (Phila Pa 1976) 2004;29(16):E326-32.

27. Lee DY, Shim CS, Ahn Y, Choi YG, Kim HJ, Lee SH. Comparison of percutaneous endoscopic lumbar discectomy and open lumbar microdiscectomy for recurrent disc herniation. J Korean Neurosurg Soc 2009;46(6):515-21. 
28. Li X, Hu Z, Cui J, Han Y, Pan J, Yang M, et al. Percutaneous endoscopic lumbar discectomy for recurrent lumbar disc herniation. Int J Surg 2016;27:8-16.

29. Yao Y, Zhang H, Wu J, Liu H, Zhang Z, Tang Y, et al. Minimally Invasive Transforaminal Lumbar Interbody Fusion Versus Percutaneous Endoscopic Lumbar Discectomy: Revision Surgery for Recurrent Herniation After Microendoscopic Discectomy. World Neurosurg 2017;99:89-95.

30. Hoogland T, Schubert M, Miklitz B, Ramirez A. Transforaminal posterolateral endoscopic discectomy with or without the combination of a low-dose chymopapain: a prospective randomized study in 280 consecutive cases. Spine (Phila Pa 1976) 2006;31(24):E890-7.

31. Macnab I. Negative disc exploration. An analysis of the causes of nerve-root involvement in sixtyeight patients. J Bone Joint Surg Am 1971;53(5):891-903.

32. Patel MS, Braybrooke J, Newey M, Sell P. A comparative study of the outcomes of primary and revision lumbar discectomy surgery. Bone Joint J 2013;95-B(1):90-4.

33. Kim HS, Paudel B, Jang JS, Lee K, Oh SH, Jang IT. Percutaneous Endoscopic Lumbar Discectomy for All Types of Lumbar Disc Herniations (LDH) Including Severely Difficult and Extremely Difficult LDH Cases. Pain Physician 2018;21(4):E401-E8.

34. Xu J, Li Y, Wang B, Lv GH, Wu P, Dai Y, et al. Percutaneous Endoscopic Lumbar Discectomy for Lumbar Disc Herniation with Modic Changes via a Transforaminal Approach: A Retrospective Study. Pain Physician 2019;22(6):E601-E8.

35. Yao Y, Liu H, Zhang H, Wang H, Zhang C, Zhang Z, et al. Risk Factors for Recurrent Herniation After Percutaneous Endoscopic Lumbar Discectomy. World Neurosurg 2017;100:1-6.

36. Choi KC, Kim JS, Park CK. Percutaneous Endoscopic Lumbar Discectomy as an Alternative to Open Lumbar Microdiscectomy for Large Lumbar Disc Herniation. Pain Physician 2016;19(2):E291-300.

37. Liu C, Zhou Y. Comparison Between Percutaneous Endoscopic Lumbar Discectomy and Minimally Invasive Transforaminal Lumbar Interbody Fusion for Lumbar Disc Herniation with Biradicular Symptoms. World Neurosurg 2018;120:e72-e9.

38. Liu C, Zhou Y. Percutaneous endoscopic lumbar discectomy and minimally invasive transforaminal lumbar interbody fusion for massive lumbar disc herniation. Clin Neurol Neurosurg 2019;176:19-24.

39. Yao Y, Zhang H, Wu J, Liu H, Zhang Z, Tang Y, et al. Comparison of Three Minimally Invasive Spine Surgery Methods for Revision Surgery for Recurrent Herniation After Percutaneous Endoscopic Lumbar Discectomy. World Neurosurg 2017;100:641-7 e1.

40. Than KD, Mummaneni PV. Unilateral approach for bilateral decompression with MIS TLIF. World Neurosurg 2014;82(5):646-7.

41. Choi I, Ahn JO, So WS, Lee SJ, Choi IJ, Kim H. Exiting root injury in transforaminal endoscopic discectomy: preoperative image considerations for safety. Eur Spine J 2013;22(11):2481-7.

42. Sairyo K, Matsuura T, Higashino K, Sakai T, Takata Y, Goda Y, et al. Surgery related complications in percutaneous endoscopic lumbar discectomy under local anesthesia. J Med Invest 2014;61(3-4):2649. 
43. Bari TJ, Karstensen S, Sorensen MD, Gehrchen M, Street J, Dahl B. Revision surgery and mortality following complex spine surgery: 2-year follow-up in a prospective cohort of 679 patients using the Spine AdVerse Event Severity (SAVES) system. Spine Deform 2020.

44. Hu X, Lieberman IH. Revision spine surgery in patients without clinical signs of infection: How often are there occult infections in removed hardware? Eur Spine J 2018;27(10):2491-5.

45. Lau D, Lee JG, Han SJ, Lu DC, Chou D. Complications and perioperative factors associated with learning the technique of minimally invasive transforaminal lumbar interbody fusion (TLIF). J Clin Neurosci 2011;18(5):624-7.

46. Lee DY, Lee SH. Learning curve for percutaneous endoscopic lumbar discectomy. Neurol Med Chir (Tokyo) 2008;48(9):383-8; discussion 8-9.

47. Wang H, Huang B, Li C, Zhang Z, Wang J, Zheng W, et al. Learning curve for percutaneous endoscopic lumbar discectomy depending on the surgeon's training level of minimally invasive spine surgery. Clin Neurol Neurosurg 2013;115(10):1987-91.

48. Hadjipavlou AG, Tzermiadianos MN, Bogduk N, Zindrick MR. The pathophysiology of disc degeneration: a critical review. J Bone Joint Surg Br 2008;90(10):1261-70.

\section{Tables}

Table 1. Preoperative data in PELD group and MIS-TLIF group

\begin{tabular}{lccc}
\hline \multicolumn{1}{c}{ Indics } & PELD group & MIS-TLIF group & P Values \\
\hline Number of patients & 24 & 22 & \\
Age (years) & $49.25 \pm 13.95$ & $56.00 \pm 7.76$ & 0.10 \\
Gender: male (\%) & $14(58.33)$ & $14(63.64)$ & 0.71 \\
BMI (kg/m) & $25.37 \pm 2.72$ & $26.21 \pm 2.41$ & 0.41 \\
Married person (\%) & 91.67 & 100 & 0.51 \\
Smoking habit (\%) & 20.83 & 18.18 & 0.82 \\
Alcohol use (\%) & 4.17 & 0 & 0.33 \\
Herniation level: & $13 / 11$ & $13 / 9$ & 0.74 \\
L4-L5/L5-S1 & & 72.7 & 0.86 \\
Paramedian herniation (\%) & 75.0 & 68.2 & 0.91 \\
Migrated herniation (\%) & 66.7 & 31.8 & 0.48 \\
Modic change (\%) & 41.7 & & \\
\hline
\end{tabular}

BMI: body mass index; PELD: percutaneous endoscopic lumbar discectomy; MIS-TLIF: minimally invasive 
transforaminal lumbar interbody fusion.

Table 2. Perioperative outcomes, complications, and recurrence condition

\begin{tabular}{lccc}
\hline \multicolumn{1}{c}{ Indics } & $\begin{array}{c}\text { PELD group } \\
(\mathbf{n = 2 4 )}\end{array}$ & $\begin{array}{c}\text { MIS-TLIF group } \\
(\mathbf{n = 2 2})\end{array}$ & P Values \\
\hline Operation time (minutes) & $113.3 \pm 45.44$ & $232.50 \pm 58.49$ & 0.000 \\
Intraoperative hemorrhage (m) & $17.75 \pm 17.05$ & $245.00 \pm 132.18$ & 0.000 \\
Post-operative hospitalization (days) & $1.90 \pm 0.97$ & $5.80 \pm 2.86$ & 0.000 \\
Complication, n (\%) & $2(8.33)$ & $1(4.55)$ & 0.60 \\
Recurrence, n (\%) & $5(20.83)$ & $0(0)$ & 0.02 \\
\hline
\end{tabular}

PELD: percutaneous endoscopic lumbar discectomy; MIS-TLIF: minimally invasive transforaminal lumbar interbody fusion.

Table 3. Clinical outcomes in PELD group and MIS-TLIF group 


\section{Indics}

PELD group

$(n=24)$

\section{VAS of back pain}

Preoperative

1 months after operation

6 months after operation

12 months after operation

VAS of leg pain

Preoperative

1 months after operation

6 months after operation

12 months after operation

\section{ODI}

Preoperative

1 months after operation

6 months after operation

12 months after operation

Clinically satisfactory, n (\%)
$7.05 \pm 0.76$

$2.15 \pm 0.59^{*}$

$1.95 \pm 0.86^{*}$

$1.20 \pm 0.62 *$
$13.90 \pm 1.29 *$

$12.30 \pm 1.26^{*}$

$10.65 \pm 0.81$ *

$22(87.5)$
MIS-TLIF group

$(\mathbf{n}=\mathbf{2 2})$
0.62

0.001

0.52

$0.90 \pm 0.57$ *

0.21

$3.00 \pm 0.67 *$

$1.80 \pm 0.42 *$
0.85

0.47

0.37
0.66
$1.10 \pm 0.64^{*}$
$1.00 \pm 0.47 *$

$28.30 \pm 2.00$

0.83

$15.10 \pm 1.29 *$

0.02

$12.00 \pm 1.05^{*}$

0.52

$10.80 \pm 0.63 *$

0.61

20 (90.9)

0.92

PELD: percutaneous endoscopic lumbar discectomy; MIS-TLIF: minimally invasive transforaminal lumbar interbody fusion; VAS: visual analog scale; ODI: Oswestry disability index.

* Statistically significant difference compared with preoperative indic.

\section{Figures}



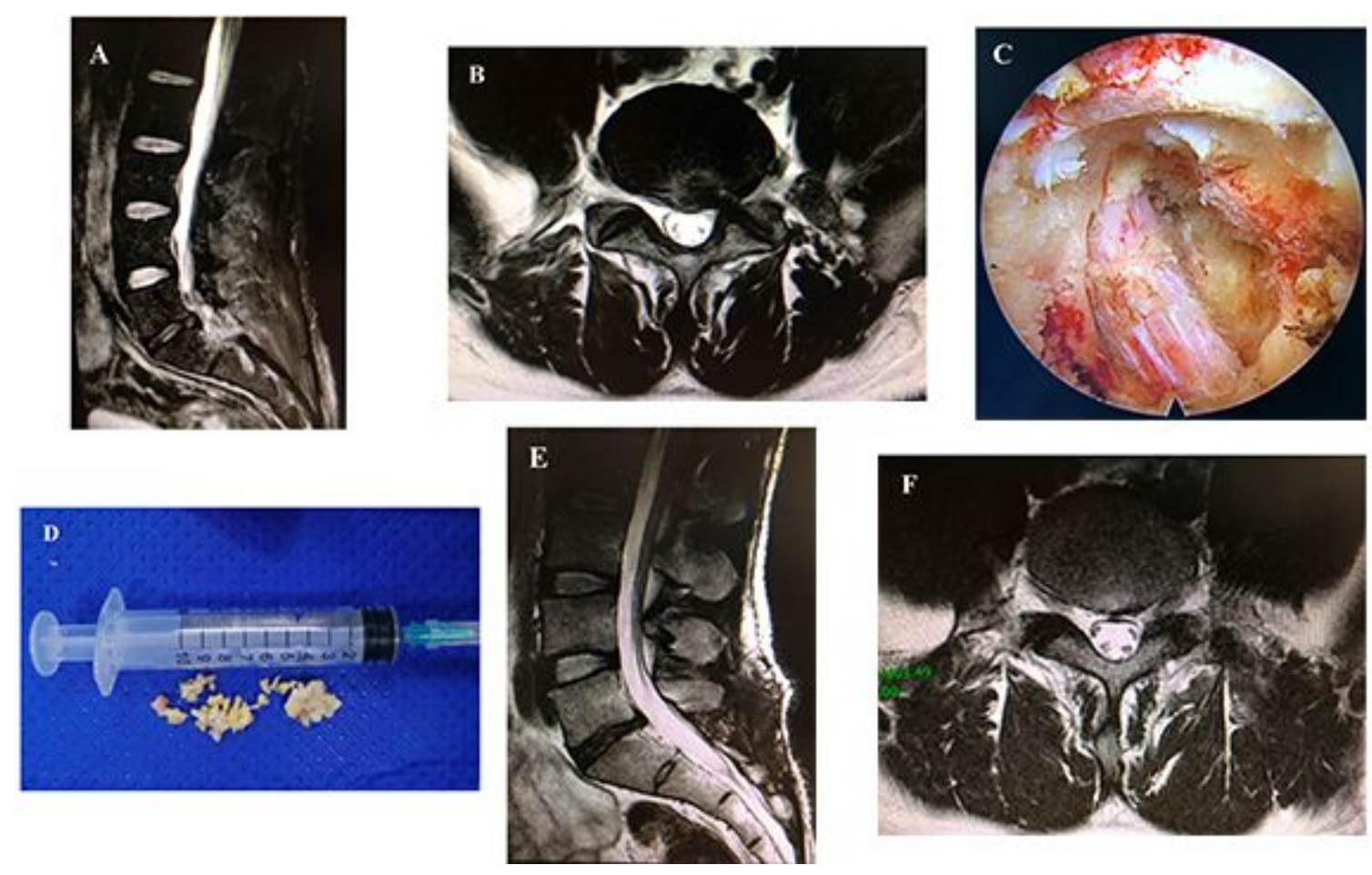

\section{Figure 1}

Pre- and postoperative MRI of a 56-year-old male with recurrent LDH who underwent revision PELD: (A) and (B) Preoperative MRI revealed recurrent disc herniation was identified on L5-S1. (C) The decompressed nerve root under endoscopic view. (D) Resected disc fragment in the revision operation. (E) and (F) Postoperative MRI showed decompressed spinal canal and foraminal area.
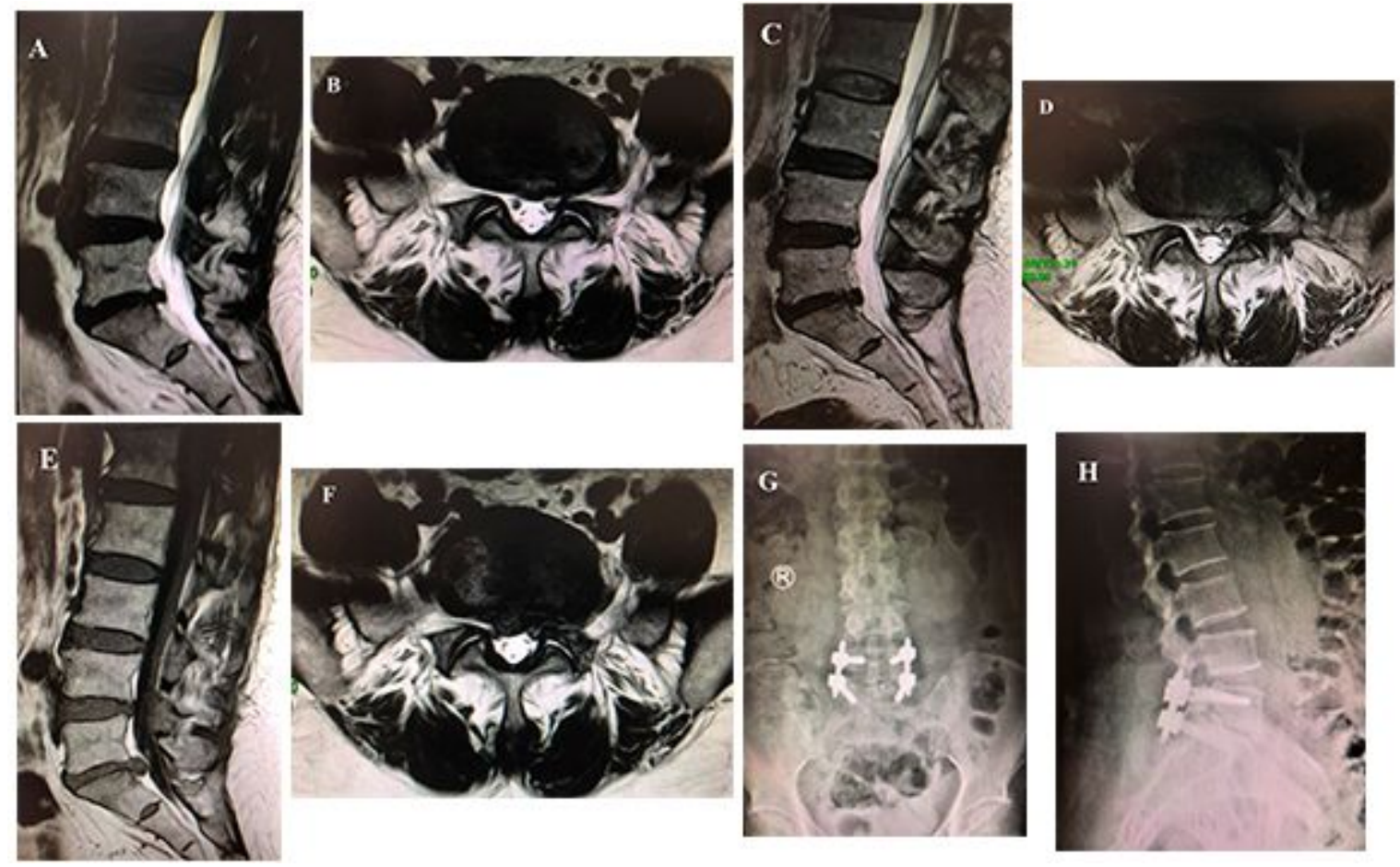

Figure 2 


\begin{abstract}
A 52-year-old female diagnosed with recurrent LDH 17 months after primary PELD and underwent revision MIS-TLIF. (A) and (B) Preoperative MRI of primary PELD revealed disc herniation was identified on L5-S1. (C) and (D) Postoperative MRI at 12 months after primary PELD showed decompressed spinal canal and foraminal area. (E) and (F) Preoperative MRI of revision MIS-TLIF at 17 months after primary PELD revealed recurrent disc herniation was identified on L5-S1. (G) Anteroposterior and (H) lateral radiographs after revision MIS-TLIF and percutaneous pedicle screw fixation.
\end{abstract}

